\title{
To Explore the Innovative Value of the Three-Pillar EAP Model for Human Resource Management in Enterprises Under the Epidemic Situation -- Take M Company as an Example
}

\author{
Yang Tian-wen ${ }^{1, \mathrm{a}}$, Luo Guangli ${ }^{2, \mathrm{~b}, *}$ \\ 1 School of Management, Nanguo Business School, Guangdong University of Foreign Studies, \\ Guangzhou, Guangdong, China \\ 2 School of Management, Nanguo Business School, Guangdong University of Foreign Studies, \\ Guangzhou, Guangdong, China \\ a2035612930@qq.com \\ b*2362404297@qq.com
}

\begin{abstract}
Abstract: In recent years, EAP began to spread widely in various enterprises in China. Many large enterprises have used EAP to achieve the goal of motivating employees and improving efficiency.Although EAP has been promoted in China for a long time, many enterprises still find EAP "not fit with the soil" and fail to make it play its role to the full.Meanwhile, as a foreign concept, the three-pillar HR model is well integrated into Chinese local enterprises.In this regard, this paper will try to integrate EAP into the HR three-pillar model to create the three-pillar MODEL of EAP, and test its effect through the practical application of M enterprises.In order to relieve the psychological pressure of employees returning to work at present, it is necessary to explore the mode of EAP better integrating into enterprises under the current epidemic situation.
\end{abstract}

Keywords: EAP, Three pillars of HR, Three pillars of EAP, Fred Luxens : Psychological Capital and performance, human resource management.

\section{探索疫情下 EAP 三支柱模式对企业人力资源管理的创新 价值研究 一一以 $\mathrm{M}$ 公司为例}

杨添文 ${ }^{1, a}$ 罗光丽 ${ }^{2, b} *$

\begin{abstract}
'管理学院, 广东外语外贸大学南国商学院, 广州, 广东, 中国 管理学院, 广东外语外贸大学南国商学院, 广州, 广东, 中国 a2035612930@qq.com

b*2362404297@qq.com
\end{abstract}

\section{摘要:}

近年来 EAP 开始盛传于我国的各个企业，很多的大企业都纷纷运用 EAP 从而达到激励员工提高效率的 目标。虽说 EAP 在中国的宣传已经有一段时间了，但是仍然很多企业对 EAP 出现 “水土不服” 的现象, 并且未能让 EAP 的作用发挥到及至。与此同时同样作为外来概念的 HR 三支柱模式却很好的融入了中国 的本土企业。对此，本文将试图把 EAP 融入到 HR 三支柱模式当中创新出 EAP 三支柱模式，并通过 M 企 业的实践运用检验其效果。力求探索出在当下疫情的情况下 EAP 更好融入企业的模式，以此缓解当下 复工企业员工的心理压力。

关键词: $E A P H R$ 三支柱 $E A P$ 三支柱 弗雷德卢森斯的心理资本与绩效 人力资源管理 


\section{1. 前言}

随着全球化的推进与我国“一带一路”政策的实施, 进一步加强了我国与国外先进思想的交流, EAP 的管理 理念及管理模式正逐渐渗入到我国。“11尤其近几年中 国经济的飞速发展, 越来越多的企业意识到员工心理健 康的重要性, EAP 先进的管理模式及科学的管理方法逐 渐受到中国诸多企业的重视。现阶段, 虽然 EAP 的引入 并应用已见成效, 但 EAP 在中国仍处于 “舶来品” 的状 态, 仍是拿来主义, 其效度仍然不高。国内学者对 EAP 运用的研究主要集中在其定义、适用范围、运用形式等 方面, 仍然未形成体系化的研究。而在我国企业 EAP 运 用管理上, 更多情况下是直接引入国外的 EAP 体系, 实 践上仍是停留在表面, 并未针对国内企业具体情况及员 工心理健康程度进行调整, 因此 EAP 的运用针对性不足。

当下，疫情蔓延全球，人们对疫情的担忧，复工后 对感染的恐惧以及担心疫情下企业无法重新运营等种 种负面情绪包裹着复工后的员工。在疫情下员工心理问 题越来越突出, 企业中的员工不仅要面对工作原有的压 力, 而且还要面对疫情下, 对人与人接触, 公共场合活 动所带来的感染风险的恐惧。以及环境冷清, 很多企业 倒闭, 随时失业的压力。在当下让 EAP 分布在企业的每 个部门, 让员工想咨询就能咨询, 有心理问题的员工第 一时间被发现并处理, 这样才能真正发挥 EAP 的作用, 真正达到促进企业复工后顺利发展的效果。现阶段我国 EAP 的运用体系化程度不足, 尤其在应用方法上仍然不 够灵活, 未能够基于我国企业具体制定体系化的 EAP 运 用。 ${ }^{[21}$ 国家安全生产监督管理局的金否夫先生曾在一 次 EAP 的交流会议中公布了这样的数据: 我国每天会产 生 5000 个职业病人，而企业界每天要为他们支付 2000 万元人民币的损失。而对 IT 行业 2000 多名员工所做的 调查表明, 有 $20 \%$ 的企业员工压力过高, 至少有 $5 \%$ 的员 工心理问题较严重。有 $75 \%$ 的员工认为他们需要心理帮 助。所以, 现阶段如何基于我国企业具体内部情况进行 EAP 运用的调整, 是极为迫切的需求。

\section{2. 研究意义}

\section{1 理论意义}

本文试图完善现有的人力资源管理模式, 在分析当 前人力资源管理弊端及 EAP 服务在中国企业实施现状 的基础上, 从弗雷德卢森斯的心理资本与绩效的关系来 分析目前疫情下影响企业员工生产力提高的原因和企 业导入 EAP 的实际问题。【3】进而参考戴维 - 尤里奇 教授 (Dave Ulrich) 提出了 HR 三支柱模式的早期理 论, 论述将 EAP 融入 HR 三支柱当中, 产生 EAP 三支柱: EAPBP (员工帮助计划业务合作伙伴); EAPSSC (员工 帮助计划共享服务中心); EAPCOE（员工帮助计划专家 中心) 的实践价值, 并分析提升企业 EAP 工作的新途径,
也将拓宽企业人力资源管理思路和维度。

\section{2 应用价值}

如今阿里巴巴的加班夜宵、腾讯的分时福利、网易 的健身室、bilibili 的游戏厅等这些无不是在关心员 工, 让员工对公司有家的感觉, 喜欢来上班, 愿意努力 工作。所以事实证明, 哪个企业能切实关心员工, 能激 发出员工的工作热情, 那么这个企业就有可能壮大、走 远。在过去, 我们知道 HR 三支柱理论惠及很多公司,

其中就有国内比较出名的腾讯公司。HR 三支柱理论之所 以受国内企业欢迎, 就是因为它符合中国企业情况而且 覆盖面广、针对性高。那么将 EAP 融入原有的 HR 三支 柱模式当中，可以让 EAP 充分拥有 HR 三支柱，覆盖面 广、针对性高而且符合中国企业情况的优势的同时利用 原有的 HR 三支柱模式可以减少再建部门出现的部门臃 肿和投入费用高的情况。利用 EAP 三支柱模式可以真正 实现 EAP 渗透进公司的每个角落惠及每个员工，激励每 个员工。

\section{3 国内外研究情况}

\subsection{1 国内研究情况}

目前 EAP 服务导入中国企业大多参照的是国外的 实施办法, 国内 EAP 服务的创新鲜有可借鉴的经验, 以下是国内专家对 EAP 服务的研究成果。【4】服务内 容上台湾专家杜桂碧将 EAP 服务内容分为五大类: 第 一, 心理咨询辅导。第二, 教育成长。第三, 文娱休闲 类。第四, 医疗保健类。第五, 福利。【5】张西超认 为 $\mathrm{EAP}$ 服务, 主要内容有压力管理、情绪管理、危机 干预、生涯规划、协助理财、法律纠纷、健康养生等。

【6】张毛龙、陈红认为所有影响组织和员工绩效的因 素都可以纳入 EAP 服务的范畴。虽然许多专家已经意 识到 EAP 服务的外延广阔, 但现行企业中的 EAP 服务 因为没有一个完善且有针对性的体系, EAP 难以渗透进 每一个部门, 方法上也多是采用针对问题员工的心理健 康状况展开普查和简单的表层形式。

\subsection{2 国外情况}

目前，美国的 500 强企业 100\%使用 EAP，100 500 员工规模的企业 70\%使用 EAP。EAP 专业人员从 60 年代的不足 20 人发展到 7000 人。据统计, $85 \%$ 以上的世界 500 强企业引入了 EAP 管理模式, 并将 之作为管理员工心理健康的主要模式和方法。【7】康 菲石油公司引入 EAP 后发现, 企业员工能举例及工作 效率得到了较为明显的改善, 员工心里疾病患有率得到 显著下降, 整体员工工作氛围较为健康。这表明 EAP 只 需要融入一个符合实施地企业的内部情况体系便可以 
发挥出不可估量的价值。而三支柱模式在中国经历时间 的检验, 它十分符合国内企业的发展状况。EAP 只需要 融入到三支柱模式当中创新为 EAP 三支柱模式便可以发 挥在国外发挥的同等价值甚至更好。

\section{4 研究内容}

本文按照提出问题、分析问题、解决问题的研究思 路, 首先说明 EAP 的概念、研究现状, 其次, 分析企 业为什么要将 EAP 融入到三支柱模式当中, 最后, 如何 建立 EAP 三支柱服务模式, 借助 $M$ 企业导入 EAP 三支柱 模式的实践案例, 总结 EAP 三支柱模式导入企业的经验, 证明其可行性。

\section{5 应用模式}

【8】在处理人力资源问题或者制定（执行）人力 资源政策时, 通过 SSC 共享服务将企业各业务单元中所 有与人力资源管理有关的问题进行分类处理, 对基础性 员工共同问题统一处理, 对个别员工特殊问题进行特殊 处理。这样就可以提高企业 EAP 的工作效率, 加快对员 工问题的处理速度。【9】集体内容有: 1、员工呼叫中 心: 支持员工和管理者发起的 EAP 服务需求; EAP 流程 事务处理中心: 支持由 $\mathrm{COE}$ 发起的主流程的 EAP 事物部 分 (如: 员工心理培训、福利、知识技能培训) ; 3 、 EAPSSC 运营管理中心: 提供 EAP 质量、内控、数据、技 术 (包括自助服务) 和员工状态管理支持。EAPSSC 是 $H R$ 工作效率提升的驱动器, 其使命是为 HR 在员工心理 健康管理和提升员工绩效这一方面提供高效、高质量和 成本最佳的 EAP 共享服务。为此, EAPSSC 通常需要依托 HRSSC 服务模式来最大化工作效率。再经过 EAPBP 利用 其自身的 EAP 专业素养来调整各单位人员关系、员工心 理状态中存在的种种问题, 从而提出并整理发现的问题 交付给专业心理咨询专家或相关部门主管, 采用专业和 有效的方法更好的解决问题或设计更加合理的工作流 程, 完善所在部门的工作使工作更人性化。最后再由 EAPCOE 为各部门员工提供心理健康方面的专业咨询。同 时帮助 EAPBP 解决在各部门 EAP 工作中遇到的专业性较 强的难题, 并从专业角度协助企业制定和完善 EAP 方面 的各项措施, 指导 EAPSSC 开展服务活动。流程图如下:

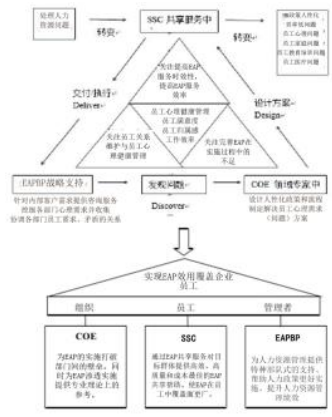

图 1 EAP 三支柱应用模式图

\section{3. 导入 EAP 三支柱模式的企业案例}

\section{3. $1 \mathrm{M}$ 企业基本情况}

M 公司是一家专注于磁性材料产品研发和制造的 国家高新技术企业，公司创办于 2004 年，坐落于浙江 省宁波市，拥有两个生产基地。秉承着 “成就客户价值” 的理念, 公司目前的产品广泛应用于曳引机、电子信息、 航空航天、汽车、医疗、国防等领域, 产品更是远销欧 美等国家, 与许多国际知名企业建立了合作伙伴关系, 在业内具有一定的影响力。作为一家民营企业, 该公 司目前拥有员工 260 人。公司注重采取自主创新战略, 注重高层次人才的引进和培养, 与宁波市多家科研院 所、大专院校建立了合作关系。并且 $\mathrm{M}$ 公司近几年在 人力资源管理方面引进了较为先进的 HR 三支柱模式。

\section{3. $2 M$ 企业导入 $E A P$ 三支柱模式的背景}

M 公司在员工关系管理中存在的问题, 大致归纳为 以下几个方面：（1）公司对员工关系管理不重视。在 $\mathrm{M}$ 公司中, 人力资源的管理日常多半是员工招聘、离职 等一些必要的事项, 对于更加细化的涉及到员工心理、 情绪等方面的需求和问题，则基本上属于放任状态。比 如在引进 EAP 三支柱模式的前一个年度, M 公司发生了 两起安全生产事件, 其中一起事件的起因就是员工因为 家庭纠纷影响, 导致工作时注意力不够集中, 情绪不稳, 在操作机器的过程中, 右脚的五个脚趾被机器切断, M 公司因此赔付了数十万的费用，给公司造成了巨大损 失，也给员工造成了永久性的伤害。

\section{3 存在的问题}

M 公司在员工关系管理中存在的问题大致归纳为 以下几个方面: (1) 公司对员工关系管理不重视。在 $\mathrm{M}$ 公司中, 有这么一个概念, 凡是人的事物都由人力资 源部解决, 所以 EAP 辐射不到除了人力资源部外的其他 部门, EAP 在 M 公司当中处于可有可无的状态。（2） 缺乏沟通。M 公司的管理人员不注重对下属各部门的员 工进行深入了解, 管理者和员工之间也缺乏一定的沟 通，导致出现问题才发现漏洞的现象时有发生。各部门 之间缺乏沟通, 上级对下级缺乏沟通, 不了解员工的工 作限度。这导致部门工作绩效下降, 而部门主管因此责 骂员工, 导致员工心理压力更大, 工作效率更低, 陷入 了这样一个死循环当中。（3）管理制度缺位。M 公司 的员工关系管理制度比较缺乏, 对既有 EAP 制度的执行 力度也不够强。EAP 的工作人员的专业性知识不够高, 只能应付一些小的心理问题。慢慢的员工对公司的心理 咨询功能不再信赖。而且 M 公司的 EAP 模式没能与时俱 进, 还是停留在照搬国外 EAP 模式的阶段, 未能更好的 融入中国员工的心理，未能符合公司的具体状况。 


\section{3. $4 M$ 公司 $E A P$ 项目需求分析}

通过对 M 公司引进 EAP 三支柱的应用模式前后员工 压力、职业关系、工作满意度等指标进行统计, 分析出 EAP 三支柱模式的实践效果, 来判断 M 企业对 EAP 三支 柱模式的需求。

\section{4.1 需求调研的组织和实施}

【10】对 $\mathrm{M}$ 公司的员工根据岗位进行分类, 将所有 抽样人员分为技术岗位 ( 10 人)、生产岗位 (121 人)、 管理岗位 ( 82 人)、财务岗位 (6 人) 、销售岗位 (12 人）、其他（5 人），共六类，总计随机选择样本 132 人。调研采取匿名的方式由人力资源部门将调研问卷分 发给不同岗位的员工, 员工根据自身实际情况及对问卷 各个问题的理解来自由的选定选项, 做完后由人力资源 部门组织问卷的回收和统计分析。在建立 EAP 三支柱模 式前发放一次问卷, 在建立 EAP 三支柱模式后再以同样 的方式再发放一次问卷。再根据两次问卷统计的数据进 行对比。具体流程如下:

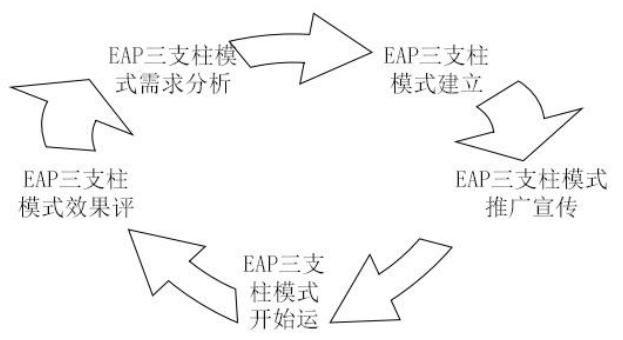

图 2 需求分析流程

\section{4. 2. 需求调研的结果分析}

对 M 公司在未建立 EAP 三支柱模式之前的员工调 查数据如下:

表 1 模式建立前调查结果

\begin{tabular}{rcccc}
\hline 问卷主题 & \multicolumn{4}{c}{ 结果说明 } \\
\hline 工作满意度 & 非常满意 $2.2 \%$ & 满意 $10.7 \%$ & 不满意 $68.9 \%$ & 不确定 $12.1 \%$ \\
职场关系 & 良好 $26.2 \%$ & 一般 $62.9 \%$ & 不确定 $7.6 \%$ & 差 $5.3 \%$ \\
压力量表 & 较大 $82.6 \%$ & 一般 $15.2 \%$ & 较小 $2.3 \%$ \\
职业规划 & 清䀿 $6.8 \%$ & 模糊 $51.5 \%$ & 短期 $40.2 \%$ \\
亲子关系 & 良好 $31.8 \%$ & 一般 $38.7 \%$ & 不好 $25 \%$ \\
\hline
\end{tabular}

从上述的调查结果分析中, 我们可以看到。 $\mathrm{M}$ 公司 原有的模式下, 员工对工作大多都是不满意的, 职场关 系也是一般, 而且 M 公司的员工大多工作压力都很大, 对职业生涯规划大多数人也是比较模糊的。在家庭的状 况方面好和不好对方半分。从这些数据我们可以看出 $\mathrm{M}$ 公司原有模式下, 企业人力资源管理是不到位的, EAP
在该企业当中并未发挥其应有的作用，员工的需求未能 得到满足。这也就是 $M$ 公司出现上述状况的一大原因。 在 EAP 三支柱管理模式建立后, 收集到的数据如下:

表 2 模式建立后调查结果

\begin{tabular}{|c|c|c|c|c|c|c|c|}
\hline 问卷内容 & & & & 结果 & & & \\
\hline \multirow{3}{*}{$\begin{array}{l}\text { 工作 } \\
\text { 满意度 }\end{array}$} & \multicolumn{2}{|c|}{ 非常满意 } & \multicolumn{2}{|c|}{ 满 意 } & \multicolumn{2}{|c|}{ 不满意 } & 不确定 \\
\hline & EAP 前 & $\mathrm{EAP}$ 后 & EAP前 & EAP扄 & $E A P$ 前 & EAP后 & EAP 前 $E A P$ 后 \\
\hline & $2.2 \%$ & $20.4 \%$ & $10.7 \%$ & $52.6 \%$ & $68.9 \%$ & $17.2 \%$ & 12. $1 \% \quad 16.7 \%$ \\
\hline & \multicolumn{2}{|c|}{ 良好 } & \multicolumn{2}{|c|}{ 一般 } & \multicolumn{2}{|c|}{ 不确定 } & 差 \\
\hline \multirow{3}{*}{ 职场关系 } & $E A P$ 前 & $\mathrm{EAP}$ 后 & EAP首 & EAP 后 & EAP 前 & $\mathrm{EAP}$ 后 & EAP前 $E A P$ 后 \\
\hline & $26.2 \%$ & $51.7 \%$ & $62.9 \%$ & $40.3 \%$ & $7.6 \%$ & $5.3 \%$ & $5.3 \%$ \\
\hline & \multicolumn{2}{|c|}{ 较大 } & \multicolumn{3}{|c|}{ 一般 } & \multicolumn{2}{|r|}{ 较小 } \\
\hline \multirow{2}{*}{ 压力状况 } & EAP 前 & $\mathrm{EAP}$ 后 & & & $\mathrm{APP}$ 后 & EAP 前 & $\mathrm{EAP}$ 后 \\
\hline & $82.6 \%$ & 49. $1 \%$ & & & $32.3 \%$ & 2. $3 \%$ & $15.6 \%$ \\
\hline
\end{tabular}

从上面的对比数据里面我们可以看出 EAP 三支柱模 式建立前后, 员工的满意度、职场关系和压力状况还是 有一定的改变的。总体来说, 员工的心理压力是在呈现 下降的趋势, 而且员工的归属感也是在加强的, 这些方 面的转变会给 M 公司的工作效率带来一定的影响, 会促 进 M 公司的发展。

\section{4. 总结}

本论文以 $\mathrm{M}$ 企业实施 $\mathrm{EAP}$ 三支柱模式为例, 证明了 EAP 三支柱模式的可行性。EAP 在国外的实践证明其可 行性与优势。在国内, EAP 的引进已经有一段时间了, 但是其对企业的效果仍然不明显。这当中有企业文化 “水土不服” 的原因，还有一个容易忽略的原因，那就 是中国与国外不仅文化有所差异, 公司的内部结构上也 有一定的差异。【11】这体现在在照搬 EAP 的前提下, 中外合资的企业把 EAP 运用的柔韧有余, 但是国内的本 土企业却难以把 EAP 真正的融入到企业当中。若 EAP 不 能融入企业当中, 这就会像一块冰放在土壤上, 如果不 能融化那么它就难以滋润一方土地。所以找到 EAP 在本 土企业中的存在形式以及系统模式显得十分的重要。经 研究发现, 同样作为 “舶来品” 的 HR 三支柱, 能够很 好的适应中国本土企业, 这当中, 腾讯、阿里巴巴等国 内著名企业都充分运用了 HR 三支柱模式。那么经思考, 若是把 EAP 与三支柱模式融合, EAP 是可以很好的融入 到国内本土企业当中, 造福国内本土企业及其员工的。 经过理论的研究以及对 $\mathrm{M}$ 公司的实践证明了, EAP 三支 柱模式是 EAP 融入本土企业的一个较优模式。而且 EAP 三支柱模式, 因为运用了三支柱模式的原理, 使 EAP 在 企业中对员工的保护更加全面、具体、专业。希望 EAP 三支柱模式能够推广开来, 惠及更多的本土企业。尤其 在当下疫情转好, 企业复工的情况下, 员工的心理状态 尤为重要, 企业能否謷过疫情, 能否在疫情后见光明这 很大部分取决于员工的归属感与工作效率。所以, 本人 希望, 在 EAP 三支柱模式得到进一步完善及检验后, 被 运用到更多的企业当中, 让企业和员工一起撑过疫情、 战胜疫情, 并在疫情过后更好的恢复生产、发展壮大。 同时 EAP 三支柱模式能得到社会的认可, 作为转换器的 
功能, 让 EAP 物尽其用惠及本土企业, 从而带动社会经 济发展。

\section{REFERENCES}

[1] Zihan Huang, Guangli Luo.2020, Research on the Value of Chinese Traditional Culture to Local EAP Model Construction.eeim. In:wuhan GB/T77142015

[2] https://www.mem.gov.cn/[Z]

[3] David Urich. Human Resource Transformation: Creating Value and Achieving Results for organizations [M]. Beijing: Electronics Industry Press, 2015.5:21

[4] Lin Gui-bi. The Rocks of The Mountains -- The Development and Current Situation of EAP in Taiwan [J]. Enterprise Research,2003(18):66-67

[5] Zhang Xichao. Effective Ways for Employee Assistance Program (EAP) to improve enterprise Performance [J]. New Economy,2003(3):57-59.

[6] Zhang Maolong, Chen Hong. Review of foreign research on EAP and Analysis of Domestic Development [J]. Enterprise Vitality, 2011(12):85-90

[7] Influence [M]. (America) Cialdini, Translated by Lu Jia. Wanjuan Publishing Co., LTD. September 2010, 33-97.

[8] Zhao Shichao. Research on the Relationship between Employee training development and Performance from the perspective of psychological Capital [D]. Shandong University of Finance and Economics,2016

[9] Zhang Guoshun, Research on Human Resource Management under the Three-pillar HR Mode of Tencent,[D], University of South China,2018, UNdefined

[10] Chen Cheng, Application research of EAP in EMPLOYEE Relationship Management of NCS Company,[D], Xiangtan University,2014, UNdefined

[11] Sun Ting, Practical Research on The Introduction of EAP Service in H Enterprises,[D], Hubei University of Technology,2018, UNdefined 